Portland State University

PDXScholar

Electrical and Computer Engineering Faculty

Publications and Presentations

Electrical and Computer Engineering

$1-1-1984$

\title{
Propagation of Airy-Hermite-Gaussian waveguide modes in free space
}

\author{
Lee W. Casperson \\ Portland State University \\ O. M. Stafsudd \\ Leroy V. Sutter \\ Jonathan Gary Grossman
}

Follow this and additional works at: https://pdxscholar.library.pdx.edu/ece_fac

Part of the Electrical and Computer Engineering Commons Let us know how access to this document benefits you.

\section{Citation Details}

Jonathan G. Grossman, Lee W. Casperson, Oscar M. Stafsudd, Leroy V. Sutter Jr. Propagation of AiryHermite-Gaussian waveguide modes in free space. Applied Optics, Volume 23, Number 1 (January 1984), pp. 48-52.

This Article is brought to you for free and open access. It has been accepted for inclusion in Electrical and Computer Engineering Faculty Publications and Presentations by an authorized administrator of PDXScholar. Please contact us if we can make this document more accessible: pdxscholar@pdx.edu. 


\title{
Propagation of Airy-Hermite-Gaussian waveguide modes in free space
}

\author{
Jonathan G. Grossman, Lee W. Casperson, Oscar M. Stafsudd, and Leroy V. Sutter, Jr.
}

\begin{abstract}
The cavity modes of metal strip waveguide lasers are most simply expressed in terms of Airy-HermiteGaussian functions. The free space propagation of the resulting beam modes has been examined, and both near- and far-field patterns have been calculated and measured. Phase plates may be useful for enhancing the far-field intensity.
\end{abstract}

\section{Introduction}

Carbon dioxide waveguide lasers are now in wide use as compact and reliable sources of $10-\mu \mathrm{m}$ laser radiation. In one important implementation the pumping is accomplished by means of a rf discharge in the lasing gas medium. ${ }^{1}$ Very recently it has been suggested that a curved metal waveguide has significant advantages over the more conventional dielectric waveguides used in most $r f$ carbon dioxide waveguide lasers, ${ }^{2,3}$ and a more thorough discussion of the relevant literature is given in Ref. 3. It must be emphasized, however, that the metal waveguide lasers produce output modes that are described by Airy-Hermite-Gaussian functions. ${ }^{2-8}$ These modes are different from the Hermite-Gaussian and Laguerre-Gaussian modes that are typical of nonwaveguide lasers, and they are also different from the modes obtained with other types of laser waveguide. While some laser applications may not be sensitive to the details of the electromagnetic field distribution, in many cases the fields must be known precisely. Unfortunately, the modes of most waveguide lasers begin to change their functional form as soon as they leave the laser, and the fields at some distant point must be obtained using free space eigenmode expansions or diffraction integrals. The purpose of this study is to investigate the propagation characteristics of the AiryHermite-Gaussian modes produced by curved metal waveguide lasers.

When this work was done all authors were with University of California, School of Engineering \& Applied Sciences, Los Angeles, California 90024; J. G. Grossman is now with Hughes Aircraft Company, Electro-Optical and Data Systems Group, El Segundo, California 90245; and L. V. Sutter is now with Directed Energy Incorporated, Irvine, California 92714

Received 1 August 1983.

0003-6935/84-010048-05\$02.00/0.

(c) 1984 Optical Society of America.
The diffraction pattern of the Airy-Hermite-Gaussian modes in free space is accurately represented by the Fresnel diffraction integral. ${ }^{2}$ Unfortunately, this integral is not soluble in terms of simple analytic functions. It is, however, easily evaluated numerically. In this paper we present the results of such numerical calculations together with experimental verification for some of the lower-order laser modes. The results of the numerical studies are presented in Sec. II with emphasis on the development of Hermite-Gaussian approximations to the Airy-Hermite-Gaussian mode functions. The advantage of these approximations is that they permit analytical calculations of the beam propagation characteristics. In Sec. III, we present experimental verification of the Gaussian model for propagation of the lowest-order Airy-Hermite-Gaussian modes.

\section{Numerical Solutions}

The basic laser waveguide structure under consideration here is shown schematically in Fig. 1. The concave and curved metal strip sketched in the figure is terminated by flat mirrors which reflect (and partially transmit) the laser mode, and the rf excited laser gas or other amplifying medium is in close proximity to the waveguide surface. To a good approximation, the fields at the output of such a laser may be regarded as plane waves having a Hermite-Gaussian amplitude distribution in the $z$ direction tangential to the waveguide surface and an Airy amplitude distribution in the $r^{\prime}$ direction normal to the waveguide surface. In mathematical terms the output fields can be written ${ }^{3}$

$$
E(\rho, \zeta)=E_{0} A(\rho) H_{m}(\zeta) \exp \left(-\zeta^{2} / 2\right)
$$

The Airy factor $A(\rho)$ is a solution of the Airy differential equation ${ }^{9}$

$$
\frac{d^{2} A}{d \rho^{2}}-\rho A=0
$$




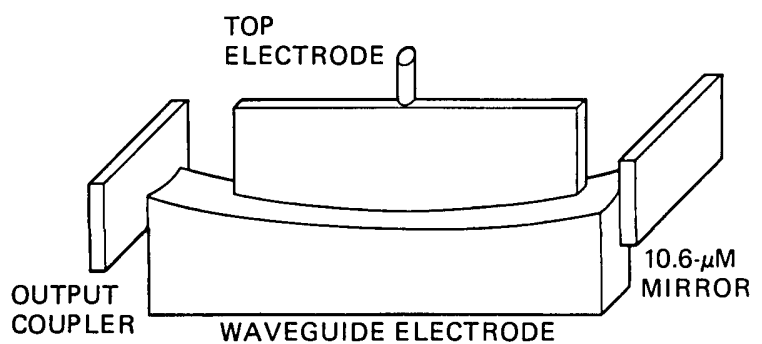

Fig. 1. Schematic representation of an $\mathrm{rf}$ excited metal waveguide laser.

where $\rho$ is the normalized and displaced radial coordinate:

$$
\rho=\left(2 k_{0}^{2} / r_{0}\right)^{-2 / 3}\left[\left(2 k_{0}^{2} / r_{0}\right) r^{\prime}-\alpha\right] .
$$

The constant $\alpha$ must be chosen so that a zero of the Airy function occurs at the waveguide surface $r^{\prime}=0$, and $k_{0}$ is the propagation constant. The Hermite factor $H(\zeta)$ is a solution of the Hermite differential equation ${ }^{10}$

$$
\frac{d^{2} H}{d \zeta^{2}}-2 \zeta \frac{d H}{d \zeta}+2 m H=0
$$

where $\zeta$ is the normalized $z$ coordinate

$$
\zeta=2^{1 / 2} z / w
$$

and $m$ is an integer denoting the transverse mode order in the $z$ direction. The spot size $w$ in Eq. (5) is related to the waveguide parameters by

$$
w=\left[2\left(r_{0} R_{0}\right)^{1 / 2} / k_{0}\right]^{1 / 2} .
$$

The Fresnel diffraction integral for the Airy-Hermite-Gaussian modes is

$$
\begin{aligned}
U\left(x_{0}, y_{0}\right)= & \frac{\exp (j k z)}{j \lambda z} \iint\left(A_{n}\left(x_{1}\right) H_{m}\left(\frac{\sqrt{2} y_{1}}{w_{0}}\right) \exp \left(\frac{-y_{1}^{2}}{w_{0}^{2}}\right)\right. \\
& \left.\times \exp \left\{j \frac{k}{2 z}\left[\left(x_{0}-x_{1}\right)^{2}+\left(y_{0}-y_{1}\right)^{2}\right]\right\}\right) d x_{1} d y_{1},
\end{aligned}
$$

where $A_{n}\left(x_{0}\right)$ is the portion of the Airy function for which $x_{0}$ is greater than the $n$th zero, $H_{m}$ is the $m$ th Hermite polynomial, and the integral is over the exit aperture of the waveguide. The diffraction integral is easily separated into an integral for a Hermite-Gaussian beam diffracting in the $y$ direction and an Airy beam diffracting in the $x$ direction. The Hermite-Gaussian integral can be evaluated analytically while the Airy integral requires numerical evaluation.

The numerical evaluation of Eq. (7) for a specific mode yields accurate near- and far-field patterns. However, this approach is limited in that it does not provide the user with any direct guidelines for design of different lasers or optical systems. Our numerical study, therefore, also attempted to develop quasi-analytical solutions for the propagation of the Airy-Hermite-Gaussian modes in free space.

The most important parameters of a laser's output mode in the far field are its beam divergence and intensity profile. As seen in Figs. 2-5, the far-field intensity profiles of the Airy-Hermite-Gaussian modes, unlike Hermite-Gaussian modes, do not retain much of their near-field properties. The beam divergences,

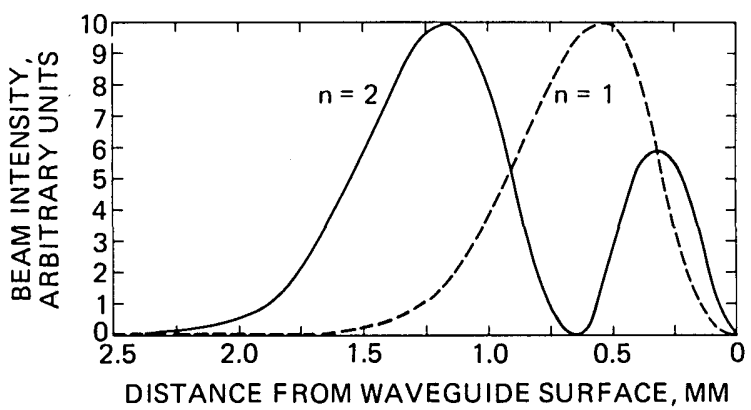

Fig. 2. Beam intensity perpendicular to the waveguide surface for the first- and second-order Airy-Gaussian modes.

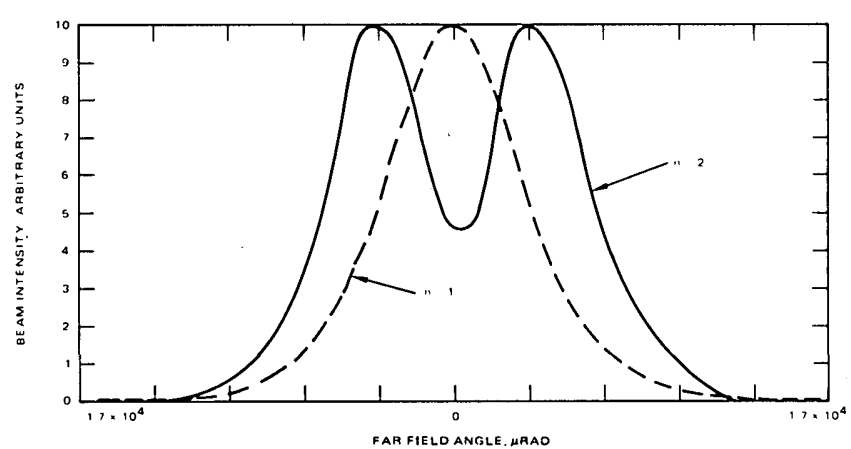

Fig. 3. Far-field beam divergence of the first- and second-order Airy-Gaussian modes.

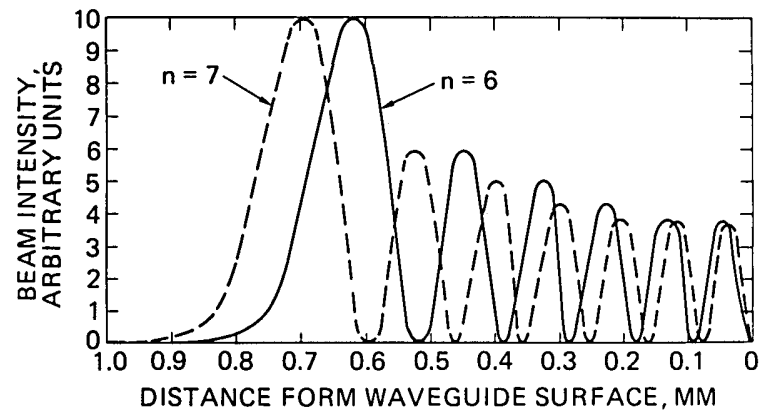

Fig. 4. Beam intensity perpendicular to the waveguide surface for the sixth- and seventh-order Airy-Gaussian modes.

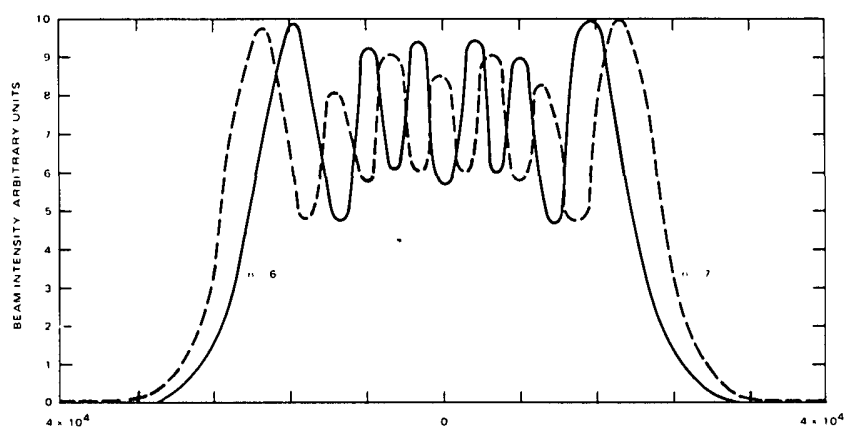

Fig. 5. Far-field beam divergence of the sixth- and seventh-order Airy-Gaussian modes. 
however, do roughly correlate to their Hermite-Gaussian counterparts. In particular, the first-order Airy mode's divergence is very close to the lowest-order Hermite-Gaussian mode $\left(\mathrm{TEM}_{00}\right)$ if the Airy mode's spot size $\Delta R$ is defined as half of the beam diameter between the $e^{-2}$ power points. It is interesting to note that for these examples the far-field intensity distribution is symmetric in spite of the large asymmetries in the near field.

The apparent similarity between the $\mathrm{TEM}_{00}$ and lowest-order Airy mode is also evident in their far-field intensity profiles. The far-field profile of the lowestorder Airy mode is accurately (less than a few percent error) represented by a Gaussian mode with the same spot size. The analogy, however, does not extend to the other beam parameters, particularly ones that are used in near-field calculations. One of the beam parameters that is significantly different for the two beams is the Rayleigh length $z_{0}$, which represents the distance the beam needs to propagate to increase its diameter by a factor of $2^{1 / 2}$. For a Gaussian beam the Rayleigh length is given by ${ }^{11}$

$$
z_{0}=\left(n \pi w_{0}^{2}\right) / \lambda,
$$

where $n$ is the index of refraction, $\lambda$ is the wavelength, and $w_{0}$ is the beam's radius to $e^{-2}$ power at the beam waist.

The propagation distance to the far field for a Gaussian beam may be regarded as roughly three Rayleigh lengths. For the Airy mode, about ten Rayleigh lengths are required before the beam starts to exhibit its far-field diffraction pattern. Similarly, the Airy mode's phase front curvature and spot size cannot be accurately approximated by the Gaussian beam parameters $R(z)$ and $w(z)$ in the near field. In the far field, however, the Gaussian beam parameters may be used to describe quite accurately the lowest-order Airy mode. These results are consistent with the propagation of other lowest-order waveguide modes such as the $\mathrm{EH}_{11}$ mode in free space. ${ }^{12}$

The higher-order Airy modes can only be represented by Hermite-Gaussian functions for order-of-magnitude calculations. This is due to the lack of symmetry exhibited by the higher-order Airy modes in the near field. The higher-order modes, as seen in Fig. 4, consist of ever narrowing maxima, each thinner and smaller than its predecessor. The nulls of the Airy function are given by ${ }^{9}$

$$
a_{s}=-f[3 \pi(4 s-1) / 8]
$$

where

$f(z) \approx z^{2 / 3}\left(1+\frac{5}{48} z^{-2}-\frac{5}{36} z^{-4}+\frac{77125}{82944} z^{-6}-\frac{108056875 z^{-8}}{6967296} \ldots\right)$,

and $a_{s}$ is the $s$ th root of the Airy function and $z$ is large. From Eq. (9) it can be shown that the maxima thickness, when defined as $\Delta_{s}=a_{s}-a_{s+1}$, decreases as the mode-order $s$ increases.

The above discussion clearly shows the difficulty of modeling Airy modes as modified Hermite-Gaussian

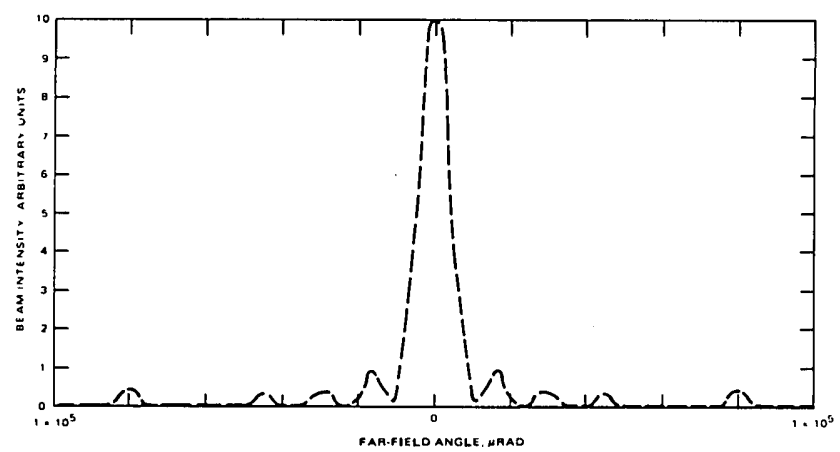

Fig. 6. Far-field beam divergence for a phase compensated seventh-order Airy-Gaussian mode.

functions. Inspection of the Airy mode's maxima, however, indicates that each maximum can be accurately represented by the Gaussian function, $\exp -\left[\left(r_{s}\right.\right.$ $\left.\left.-p_{s}\right)^{2} 4 / R_{s}^{2}\right]$, where $r_{s}$ is the perpendicular distance from the waveguide surface, $p_{s}$ is the distance to the peak of the $s$ th maximum, and $R_{s}$ is the spot size of the $s$ th maximum (distance between $e^{-2}$ power points).

The far-field diffraction of the $s$ th-order Airy mode can, therefore, be calculated by summation of the farfield diffraction of $s$ Gaussians. A more convenient formula although slightly less accurate $(\approx 10 \%)$ is

$$
\theta_{s} \text { (full angle) }=\frac{\lambda}{n \Delta_{s / 2} \pi},
$$

where $\theta_{s}$ is the far-field beam divergence of the $s$-order Airy mode and $\Delta_{s / 2}$ is the diameter of the maximum having one-half of the peak intensity of the first maximum.

The far-field diffraction of the higher-order Airy modes can be greatly enhanced by use of a phase plate. The phase plate as defined by Casperson is a transparent plate that, when inserted in the beam, forces all portions of the wave front to oscillate in phase. ${ }^{13}$ This is different from the standard situation where the electric field alternates between positive and negative values over the different nodes of the mode. It has been shown that a phase compensated beam can have a farfield peak intensity more than an order of magnitude greater than the uncompensated beam. ${ }^{13}$ A numerical evaluation of the propagation of a seventh-order Airy first-order Hermite-Gaussian phase compensated mode has been performed, and Fig. 6 shows the results. The far-field peak intensity was found to be twelve times greater for the compensated beam than for the uncompensated beam. Numerical evaluation of several other higher-order Airy modes has yielded similar results.

\section{Experiment}

The lowest-order waveguide mode is almost always the most important mode. ${ }^{12}$ This is especially true for metal waveguides where the lowest-order mode is the only mode that evolves into a near-Gaussian mode. The experimental examination of the free space propagation of this mode was, therefore, the primary goal of our study. 


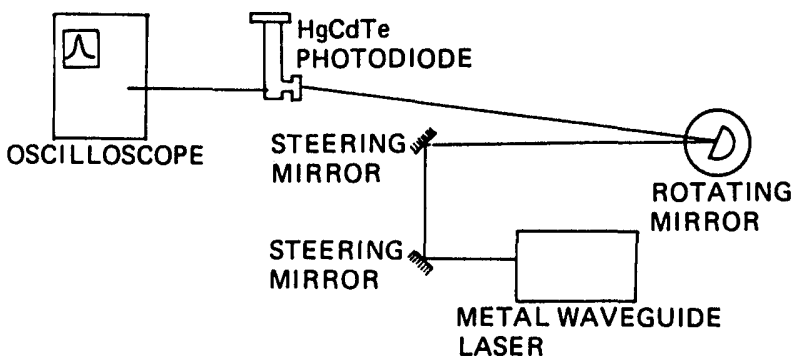

Fig. 7. Experimental setup for studying free space beam propagation.

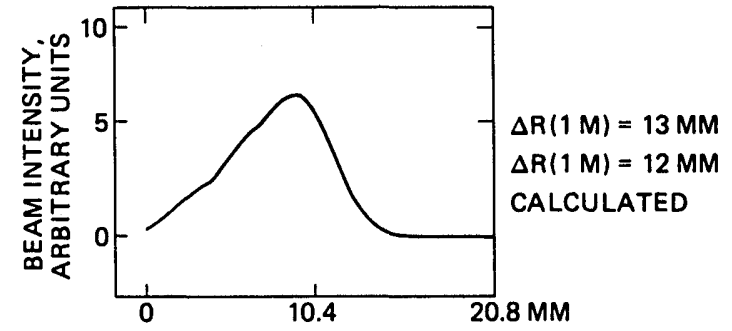

Fig. 8. Near-field beam intensity profile along Airy axis, lowest-order Airy mode ( $1 \mathrm{~m}$ from laser).

The experimental arrangement is shown in Fig. 7 . Two steering mirrors direct the metal waveguide laser output beam to the rotating mirror. The distance between the laser and rotating mirror is adjustable from 0.5 to $1.5 \mathrm{~m}$. (After $1.5 \mathrm{~m}$ the rotating mirror starts to clip the beam.) The rotating mirror assembly is mounted on a rotatable platform enabling beam scans along either the Airy or Gaussian axes of the beam. The detector is nearly collinear with the second steering mirror and the rotating mirror to minimize diffraction into the detector from the edge of the rotating mirror. The resulting scope trace is in units of beam intensity (vertical) and distance (horizontal) with the horizontal scale given by $x / \mathrm{div}=2 \Omega r t / \mathrm{div}$, where $\Omega$ is the angular frequency of the rotating mirror, $r$ is the distance from the rotating mirror to the detector, and $t /$ div is the time scale of the oscilloscope.

Beam scans were taken along the Airy axis of the beam at 1- and 3-m distance from the laser and are shown in Figs. 8 and 9. Both beam scans show a lowest-order Airy mode in close (within 10\%) agreement with the calculated results. It was found that under severe misalignment of the laser (both optics and top rf electrode tilted $\sim 1^{\circ}$ each), the laser output is a second-order transverse Airy mode as shown in Fig. 10. Only a few beam scans were taken along the Gaussian axis since the propagation of Gaussian modes in free space is well known. Figure 11 shows a scan taken $1 \mathrm{~m}$ from the laser, and the spot size in this scan is also in excellent agreement with the theory.

\section{Conclusion}

The propagation of Airy-Hermite-Gaussian beams in free space has been examined both theoretically and experimentally. The lowest-order Airy-Gaussian mode has been shown theoretically to evolve into a Gaussian beam in the far field, while the higher-order modes evolve into more complex beams. The far-field beam divergence of all Airy-Hermite-Gaussian modes, however, can be related to a first-order Gaussian approximation to the Airy function. The use of a phase plate enhances the peak far-field beam intensity of the higher-order modes by an order of magnitude or more. Our experimental results verified the near-field calculations for the first- and second-order Airy modes as well as the far-field calculations for the lowest-order AiryGaussian mode.

The results of this study indicate that the lowestorder Airy-Gaussian mode propagates in free space in a manner similar to other lowest-order waveguide

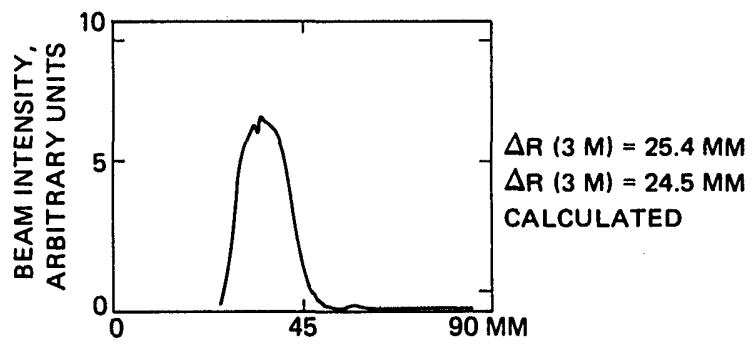

Fig. 9. Far-field beam intensity profile along Airy axis, lowest-order Airy mode ( $3 \mathrm{~m}$ from laser).

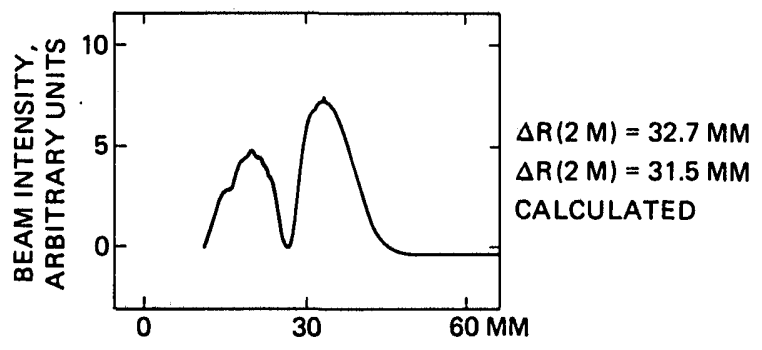

Fig. 10. Near-field beam intensity profile along Airy axis, secondorder Airy mode (2 $\mathrm{m}$ from laser).

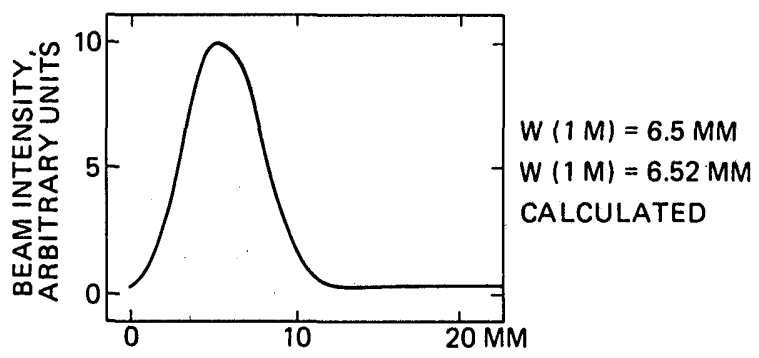

Fig. 11. Far-field beam intensity profile along Hermite-Gaussian axis, lowest-order Hermite-Gaussian mode ( $1 \mathrm{~m}$ from laser). 
modes. For practical applications, it is, therefore, possible to replace conventional hollow dielectric waveguide lasers with concave metal waveguide lasers. The primary advantages of such a replacement would be lower fabrication cost and potential higher power capabilities of the metal waveguide lasers.

The authors gratefully acknowledge D. Fink's help in the numerical computations and many useful discussions with F. G. Allen, M. F. Nicol, and R. A. Satten.

\section{References}

1. K. D. Laakman, U.S. Patent 4,169,251 (1979).

2. J. G. Grossman, "RF Excited $\mathrm{CO}_{2}$ Metal Waveguide Laser," Ph.D. Dissertation, U. California, Los Angeles (Mar. 1982).
3. J. G. Grossman, L. W. Casperson, and O. M. Stafsudd, Appl. Opt. 22, 1298 (1983).

4. M. E. Marhic, L. I. Kwan, and M. Epstein, Appl. Phys. Lett. 33, 609 (1978).

5. M. E. Marhic, L. I. Kwan, and M. Epstein, Appl. Phys. Lett. 33, 874 (1978).

6. L. W. Casperson and T. S. Garfield, IEEE J. Quantum Electron. QE-15, 491 (1979).

7. M. E. Marhic, J. Opt. Soc. Am. 69, 1218 (1979).

8. M. E. Marhic, L. I. Kwan, and M. Epstein, IEEE J. Quantum Electron. QE-15, 487 (1979).

9. Handbook of Mathematical Functions, NBS Applied Math. Ser. 55 (U.S. GPO, Washington, D.C., 1964), Chap. 10.

10. In Ref. 9, Chap. 22.

11. A. Yariv, Quantum Electronics (Wiley, New York, 1975), p. 111.

12. R. L. Abrams, "Waveguide Gas Lasers," in Laser Handbook (North-Holland, Amsterdam, 1979), Chap. A2.

13. L. W. Casperson, Opt. Quantum Electron. 8, 537 (1976).
COURSE TITLE:

COURSE TOPICS:

CONTACT:

COURSE LENGTH:

Cost: $\cdot \$ 800.00$

DATES/LOCATION: February 13-17, 1984 (Dallas, TX)
5 Days

ENGINEERING TECHNOLOGY, INC.

P.O. Box 8859

Waco, TX 76714

(817) $772-0082$

Basic elements of a laser, power and wavelength measurements, optical amplifiers and gain, three and four level lasers, optical cavity and mode structure, characteristics of laser mirrors, optical cleaning methods, laser calculation workshops, analysis of seven specific lasers and survey of commercial laser systems.

\section{CONTINUING EDUCATION UNITS: 3 CEU S}

